\title{
The effect of clinical hierarchical nursing intervention on patients with acute non-variceal upper gastrointestinal bleeding
} Qin Shen ${ }^{1, \star}$, Yang Zheng ${ }^{1}$, Ting $\mathrm{He}^{1}$, Ting $\mathrm{Mao}^{1}$

${ }^{1}$ Department of Gastroenterology, The 904th Hospital of Joint Logistic Support Force, PLA, 214000 Wuxi City, Jiangsu Province, China

\section{*Correspondence} qq_ss12@163.com (Qin Shen)

\begin{abstract}
Introduction: Acute non-variceal upper gastrointestinal bleeding has high risk of rebleeding, severely affecting patients' life. While this risk of rebleeding can be effectively reduced via targeted clinical hierarchical nursing intervention.

Object: To investigate the effect of clinical hierarchical nursing intervention on patients with acute non-variceal upper gastrointestinal bleeding (ANVUGIB).

Design: Randomized controlled study.

Methods: A total of 150 ANVUGIB patients treated in the Department of Gastroenterology in our hospital from January 2017 to October 2020 were selected and equally divided into two groups according to random number table method. The control group received routine care for ANVUGIB, including routine health education, health monitoring, medication care, etc. The nursing staff in the intervention group were divided into four different levels: senior, intermediate, junior and assistant nursing staff, and they were assigned with different responsibilities and nursing contents. The baseline conditions of the two groups of patients were compared, and the evaluation indexes include: incidence of bleeding-related complications during hospitalization, rebleeding situation within 1 month after discharge, quality of life, medical compliance, mastery rate of disease knowledge, comfort level, and nursing satisfaction.

Results: There was no significant difference in the basic condition between the two groups $(p>0.05)$, showing comparability. The incidence of complications and rebleeding during hospitalization in the observation group were both $4.00 \%$, which were significantly lower than those in the control group $\left(\chi^{2}=6.996,4.127, p<\right.$ $0.05)$. After nursing intervention during hospitalization, the scores of physiological function, physiological functioning, body pain, general health, vitality, social function, mental health and emotional function of ANVUGIB patients in the observation group were significantly higher than those in the control group at discharge $(p<0.05)$. In addition, the mastery rate of disease knowledge, comfort level and nursing satisfaction of ANVUGIB patients in the observation group were significantly higher than those in the control group $(p<0.05)$. Although the compliance rate in the observation group was higher than that in the control group, no significant difference was found $(p>0.05)$.

Conclusion: Clinical nursing intervention can significantly reduce the incidence of complications and rebleeding in patients with acute non-variceal upper gastrointestinal bleeding, and improve their quality of life, comfort level and nursing satisfaction.
\end{abstract}

\section{Keywords}

Hierarchical nursing; Acute non-variceal upper gastrointestinal bleeding; Rebleeding; Quality of life; Compliance

\section{Introduction}

Acute non-variceal upper gastrointestinal bleeding (ANVUGIB) refers to the bleeding of upper digestive tract located above the Treitz ligament due to non-variceal disease, mainly involving the pancreatic duct or bile duct and the vicinity of the anastomosis after gastrojejunostomy [1]. The etiology of ANVUGIB is complicated, and common causes include peptic ulcer, gastrointestinal malignancy and acute gastric mucosal lesions [2, 3]. ANVUGIB has a high rebleeding rate, up to $15 \%$, and a fatality rate of about $5 \%$ to $10 \%[4,5]$, which seriously threatens the life of patients. Studies have shown that improper care is also one of the important reasons for the increasing risk of rebleeding and affecting the effect of hemostasis. Targeted clinical hierarchical nursing intervention can effectively reduce the 
risk of rebleeding in patients. Hierarchical nursing is an emerging nursing mode, and its nursing process is more standardized and efficient, and the quality of nursing is effectively improved, comparing to the traditional nursing mode [6]. At present, the hierarchical nursing mode has been applied to cervical cancer, general surgery and other fields, and it has been proved that it can effectively reduce the risk of rebleeding in patients with cerebral hemorrhage caused by high cerebral blood pressure through standardized nursing management, which is worthy of promotion. Therefore, in this study, patients with ANVUGIB were intervened by clinical hierarchical nursing mode to further explore its intervention effects and provide theoretical references for clinical promotion.

\section{Objects and methods}

\subsection{Research objects}

A total of 150 ANVUGIB patients who were treated in the Department of Gastroenterology in our hospital from January 2017 to October 2020, were selected as the research objects, including 105 males and 45 females with an average age of $63.26( \pm 10.91)$ years old. According to the random number table method, the research objects were randomly divided into the observation group and the control group, with 75 patients in each group.

Inclusion criteria: (1) All patients underwent endoscopic and vomit or fecal occult blood test detection, which was in line with the ANVUGIB diagnosis in the "Guidelines for the Diagnosis and Treatment of Acute Non-Variceal Upper Gastrointestinal Bleeding" [4]; (2) All patients voluntarily participated in the research and have signed the informed consent.

Exclusion criteria: (1) Patients with variceal upper gastrointestinal bleeding, or bleeding in parts other than the upper gastrointestinal tract; (2) Patients who died during hospitalization with acute massive bleeding; (3) Patients with severe mental illness or cognitive impairment; ICU and IMCU patients were excluded.

All procedures performed in studies involving human participants were in accordance with the standards upheld by the Ethics Committee of the 904th Hospital of Joint Logistic Support Force, PLA and with those of the 1964 Helsinki Declaration and its later amendments for ethical research involving human subjects (Approval No. 2015-07).

\subsection{Nursing methods}

The control group was given routine care, and all the nursing staff mastered surgical procedures, worked closely with the doctors to complete related treatments, successfully finished hemostasis, paid close attention to the patients' changes in clinical signs including blood pressure, blood oxygen saturation and heart rate, and observed mental status changes and symptoms of hemorrhagic peripheral circulatory failure. According to the clinical doctors' advice, the nursing staff provided timely medication care of dextrose saline, balance solution, or other plasma substitutes, recorded the patient's water intake and output, color and nature of vomit and stool, etc., regularly examined blood routines to know the anemia status, carried out routine health education about the disease knowledge, and provided scientific guidance of diet. Types of medications taken by patients include anticoagulants, steroids, and nonsteroidal anti-inflammatory drugs. The observation group was given hierarchical nursing intervention, mainly includes: (1) Nursing staff level setting: the responsible nursing staff were divided into four different levels: senior, middle-level, primary and assistant levels, according to their educational background, work ability and experience. (2) Nursing staff training: nursing knowledge training and assessment were carried out to the nursing staff at each level according to their job responsibilities. (3) Duty division and care content of nursing staff at different levels: nursing teams were formed by nursing staff at different levels according to a certain proportion, and each nursing team had a senior nursing staff as the team leader, responsible for the group's nursing work arrangement. The assistant nursing staff were responsible for helping patients with the anal skin and surgical wounds disinfection, instructing patients to use light salt water for oral care, periodically collecting venous blood samples for examination following doctors' requirements, and patients' comfort level and satisfaction surveys. The primary nursing staff were responsible for the medication care to the patients including rehydration, fluid infusion, and blood enrichment in accordance with the doctor's advice. They were also responsible for psychological care, i.e., communicating with the patients with a positive, optimistic and gentle attitude, to relieve the patients' nervousness, anxiety, fear and other negative emotions, and help the patients build confidence of recovery. If the patients were found rebleeding, infection, hemorrhagic shock, suffocation, pyloric obstruction, peritonitis, or any other emergent situation, the primary nursing staff were responsible for immediately reporting the situation to the middle-level nursing staff and clinicians. At the same time, they were also responsible for the patients' compliance investigation. The middle-level nursing staff were responsible for establishing two or more intravenous channels for the patients to facilitate the primary nursing staff to carry out rehydration, fluid infusion, blood enrichment and other medication care. They were also responsible for monitoring the patients' blood pressure, blood oxygen saturation, heart rate and other vital signs, observing the color and amount of the patients' drainage fluid, and reporting emergent situations, such as rebleeding, infection, hemorrhagic shock, suffocation, pyloric obstruction and peritonitis, to the senior nursing staff and clinicians. Moreover, they were responsible for popularizing health knowledge about upper gastrointestinal hemorrhage to the patients, and investigating patients' mastery of disease knowledge. The senior nursing staff were responsible for participating in emergency treatment and nursing of the patients with rebleeding, hemorrhagic shock, suffocation etc., and for evaluating and investigating the quality of the patients' life. If low-level nursing staff found any abnormal situation of the patients, they must report to a higher-level nursing staff promptly. Each nursing team implemented an 8-hour shift system, performed its own duties, and established an accountability system. At the same time, a WeChat group was set up for doctors, nurses, and patients, so that the nursing 
staff could promptly reply to the patients' questions, push the health knowledge about upper gastrointestinal bleeding to the chat group, and provide rationalized rehabilitation recommendations to the patients. (4) Quality control: each nursing team leader summarized the nursing work of the group every week, looked for problems, and promptly adjusted the nursing implementation plan accordingly.

\subsection{Observatory index}

(1) Incidence of bleeding-related complications during hospitalization, such as infection, hemorrhagic shock, suffocation, pyloric obstruction, and peritonitis were recorded. (2) The scores of quality of life (QOL) between the two groups after nursing intervention were evaluated and compared using the Quality of Life Scale (SF-36) [5]. There are eight dimensions and 36 items of the evaluation, including physiological function, physiological functioning, physical pain, general health, vitality, social function, mental health, and emotional function. Each dimension was finally converted to a full score of 100 points according to its weight. The higher the score, the higher the patient's quality of life. (3) After nursing intervention, the compliance of the two groups with medical care was investigated and compared using the self-made medical care compliance questionnaire with a total score of 100 points. Scoring more than 80 points was regarded as compliance (medical care compliance $=$ number of compliance $/$ total number of patients $\times 100 \%$ ). (4) After nursing intervention, the disease knowledge mastery rates of the two groups were compared using self-made disease knowledge mastery rate survey table with a total score of 100 points. Scoring more than 80 points was regarded as mastered (disease knowledge mastery rate $=$ number of mastered / total number of patients $\times 100 \%$ ). (5) Comparison of comfort level of the two groups during hospitalization was conducted at discharge using self-made comfort level questionnaire. The questionnaire contains 28 items, and finally converted to a full score of 100 points according to its weight. (6) Comparison of the nursing satisfaction of the two groups during the hospitalization was carried out at discharge using self-made medical care satisfaction questionnaire with a total score of 100 points. Scoring more than 90 points was regarded as satisfied, scoring between 70 and 90 points was regarded as generally satisfied, and scoring less than 70 points was regarded as unsatisfied. Nursing satisfaction $=$ (number of satisfied patients + number of generally satisfied patients) / total number of patients $\times 100 \%$.

\subsection{Statistical analysis}

The SPSS 19.0 statistical software package was used for statistical analysis. The counting data was expressed by the number of cases (percentage), and the comparison was made by $\chi^{2}$ test. The measurement data was expressed by the mean \pm standard deviation $(\bar{x} \pm \mathrm{s})$, and the comparison was made by $t$ test. $p<$ 0.05 means the difference is statistically significant.

\section{Results}

\subsection{General information of the research objects}

Statistical analysis showed that there were no statistical differences in gender, age, course of disease or type of disease between the two groups (Table $1, p>0.05$ ).

\subsection{The incidence of complications and rebleeding during hospitalization}

As shown in Table 2, this study found that the incidence in the observation group was $4.00 \%(3 / 75)$, while the incidence of rebleeding in the control group was $13.33 \%(10 / 75)$ and the incidence of complications was $17.33 \%(13 / 75)$. Statistical analysis showed that the incidence of rebleeding and total complications in the observation group were significantly lower than those in the control group $(p<0.05)$.

\subsection{Comparison of quality of life between the two groups}

As shown in Table 3, the study found that before nursing intervention, there was no statistical difference in the scores of eight dimensions (physiological function, physiological functioning, physical pain, general health, vitality, social function, mental health, and emotional function) between the two groups $(p>0.05)$. After nursing intervention, the scores of eight dimensions of the observation group were significantly higher than those of the control group $(p<0.05)$.

\subsection{Comparison of medical compliance and disease knowledge mastery rate between the two groups}

The study found that the medical compliance of the observation group and the control group were $93.33 \%$ (70/75) and 88.00\% (66/75), respectively. The value of the observation group was higher than that of the control group, but there was no significant difference after statistical analysis $\left(\chi^{2}=1.261\right.$, $p=0.262$ ). The disease knowledge mastery rates of the observation group and the control group were $90.67 \%$ (68/75) and $70.66 \%(53 / 75)$, and the value of the observation group was significantly higher than that of the control group $\left(\chi^{2}=\right.$ 9.618, $p=0.002$ ).

\subsection{Comparison of patients' comfort level between the two groups}

In this study, it was found that the comfort level of the observation group was $82.29( \pm 10.88)$ points, which was significantly higher than that of the control group (77.09 \pm 12.24 points) $(t$ $=2.750, p=0.007)$.

\subsection{Comparison of nursing satisfaction between the two groups}

As shown in Table 4, the study found that the nursing satisfaction of the observation group was $98.66 \%$, and that of the control group was $89.33 \%$. According to Fisher's accurate test, there was a statistical difference in nursing satisfaction between the two groups $(p<0.05)$. 
TA B L E 1. General information of the research objects.

\begin{tabular}{|c|c|c|c|c|c|c|c|c|c|c|c|c|c|}
\hline \multirow[t]{2}{*}{ Group } & \multirow{2}{*}{$\begin{array}{l}\text { Gender } \\
(\mathrm{M} / \mathrm{F})\end{array}$} & \multirow{2}{*}{\multicolumn{2}{|c|}{$\begin{array}{l}\text { Age } \\
\text { old) }\end{array}$}} & \multicolumn{5}{|c|}{ Comorbidities } & \multicolumn{5}{|c|}{ Type of disease } \\
\hline & & & & Diabetes & Hypertension & $\begin{array}{l}\text { Chronic renal } \\
\text { insufficiency }\end{array}$ & $\begin{array}{l}\text { Coronary } \\
\text { heart } \\
\text { disease }\end{array}$ & Others & $\begin{array}{l}\text { Esophageal- } \\
\text { cardiac mucosal } \\
\text { tear syndrome }\end{array}$ & $\begin{array}{l}\text { Gastric } \\
\text { l cancer }\end{array}$ & $\begin{array}{l}\text { Esophageal } \\
\text { cancer }\end{array}$ & Peptic ulcer & $\begin{array}{l}\text { Acute gastric } \\
\text { mucosa } \\
\text { inflammation }\end{array}$ \\
\hline $\begin{array}{l}\text { Observation } \\
\text { group }(n=75)\end{array}$ & $53 / 22$ & $62.07 \pm 11.15$ & $5.03 \pm 0.40$ & 12 & 19 & 10 & 15 & 19 & 12 & 28 & 11 & 15 & 9 \\
\hline $\begin{array}{l}\text { Control group } \\
(\mathrm{n}=75)\end{array}$ & $52 / 23$ & $64.44 \pm 10.58$ & $5.01 \pm 0.47$ & 13 & 14 & 13 & 22 & 13 & 10 & 26 & 17 & 15 & 7 \\
\hline$\chi^{2}$ value & 0.125 & 1.334 & 0.244 & & & 3.638 & & & & & 1.792 & & \\
\hline$p$ value & 0.723 & 0.184 & 0.807 & & & 0.657 & & & & & 0.774 & & \\
\hline
\end{tabular}

TA B L E 2. Complications and rebleeding occurred in both groups during hospitalization [n (\%)].

\begin{tabular}{lcccccc} 
Group & Rebleeding $(\mathrm{n})$ & \multicolumn{4}{c}{ Bleeding-related complications } \\
& & Infection & Hemorrhagic shock & Asphyxia & Pyloric obstruction & Peritonitis \\
Observation group $(\mathrm{n}=75)$ & $3(4.00)$ & $1(1.33)$ & $1(1.33)$ & $0(0)$ & $1(1.33)$ & $0(0)$ \\
Control group $(\mathrm{n}=75)$ & $10(13.33)$ & $3(4.00)$ & $3(4.00)$ & $1(1.33)$ & $4(5.33)$ & $2(2.67)$ \\
$\chi^{2}$ value & 4.127 & & & 6.996 & 0.008 & \\
$p$ value & 0.042 & & & &
\end{tabular}

TA B L E 3. Comparison of quality of life between the two groups $(\bar{x} \pm s)$.

\begin{tabular}{|c|c|c|c|c|c|c|c|c|c|}
\hline Group & Time & Physiological function & Physiological functioning & Body pain & Overall health & Activity & Social function & Mental health & Emotional function \\
\hline \multirow[t]{2}{*}{$\begin{array}{l}\text { Observation } \\
\text { group }(\mathrm{n}=75)\end{array}$} & $\begin{array}{l}\text { Before } \\
\text { treatment } \\
(\mathrm{T} 1)\end{array}$ & $54.88 \pm 15.53$ & $52.44 \pm 17.31$ & $54.97 \pm 17.50$ & $51.71 \pm 17.18$ & $53.05 \pm 15.77$ & $45.07 \pm 14.75$ & $45.62 \pm 14.61$ & $51.25 \pm 15.30$ \\
\hline & $\begin{array}{l}\text { After treatment } \\
\text { (T2) }\end{array}$ & $85.81 \pm 9.08$ & $84.72 \pm 10.33$ & $82.17 \pm 11.65$ & $81.85 \pm 13.11$ & $78.93 \pm 12.20$ & $778.37 \pm 11.26$ & $81.48 \pm 13.86$ & $81.61 \pm 12.91$ \\
\hline \multirow[t]{2}{*}{$\begin{array}{l}\text { Control } \\
(n=75)\end{array}$} & $\begin{array}{l}\text { Before } \\
\text { treatment } \\
\text { (T3) }\end{array}$ & $56.51 \pm 14.10$ & $53.80 \pm 15.86$ & $53.87 \pm 16.08$ & $53.38 \pm 16.17$ & $57.25 \pm 19.23$ & $46.77 \pm 16.43$ & $44.52 \pm 15.52$ & $50.68 \pm 15.39$ \\
\hline & $\begin{array}{l}\text { After treatment } \\
\text { (T4) }\end{array}$ & $80.48 \pm 10.21$ & $79.61 \pm 11.98$ & $76.97 \pm 13.45$ & $75.64 \pm 13.74$ & $74.52 \pm 13.42$ & $73.40 \pm 12.93$ & $76.25 \pm 12.67$ & $76.31 \pm 13.91$ \\
\hline$t$ value/ $p$ value & T1 VS. T3 & $0.635 / 0.503$ & $0.502 / 0.617$ & $0.403 / 0.687$ & $0.617 / 0.538$ & $1.462 / 0.146$ & $0.669 / 0.504$ & $0.461 / 0.646$ & $0.228 / 0.820$ \\
\hline$t$ value/ $p$ value & T2 VS. T4 & $3.380 / 0.001$ & $2.796 / 0.006$ & $2.531 / 0.012$ & $2.832 / 0.005$ & $2.107 / 0.037$ & $0.512 / 0.013$ & $2.410 / 0.017$ & $2.554 / 0.012$ \\
\hline
\end{tabular}


TA B L E 4. Comparison of nursing satisfaction between the two groups [n (\%)].

\begin{tabular}{lcccc} 
Group & Satisfied & Generally satisfied & Unsatisfied & Satisfaction \\
Observation group $(\mathrm{n}=75)$ & $65(86.67)$ & $9(12.00)$ & $1(1.33)$ & $74(98.66)$ \\
Control group $(\mathrm{n}=75)$ & $54(72.00)$ & $13(17.33)$ & $8(10.67)$ & $67(89.33)$ \\
$p$ value & & & & 0.034 \\
\hline
\end{tabular}

\section{Discussion}

ANVUGIB is common critical and dangerous clinical disease, which is rapid in progress, prone to cause peripheral circulatory dysfunction and even the whole body function failure [6-8], bringing great threats to health and safety of the patients. In the course of the treatment, effective nursing intervention to the patients plays vital role in improving the curative effect and ensuring patient's life. The traditional nursing mode has no obvious job duty division, and the nursing staff with different qualifications, abilities and experience have basically the same working content, which leads to the situation that the nursing staff have no sense of achievement or belonging, and the nursing quality is lowered. However, and the hierarchical nursing groups the nursing staff into different levels according to their educational background, working abilities and experience, such that the job contents and responsibilities of the nursing staff at different levels are different. This type of medical care with clear job duty division provides more scientific nursing service, and popularize disease prevention knowledge and mental health care to the patients, largely improving the quality of medical care [9-11]. In this study, the effect of clinical hierarchical nursing intervention on patients with ANVUGIB was investigated.

\subsection{Clinical hierarchical nursing intervention can reduce the incidence of bleeding-related complications and rebleeding in patients with ANVUGIB during hospitalization}

This study showed that the incidence of bleeding-related complications and rebleeding in patients with ANVUGIB was significantly reduced, indicated that the hierarchical nursing was conducive to reducing the incidence of bleeding-related complications and rebleeding. In the process of hierarchical nursing intervention, targeted nursing is carried out according to the patient's condition, the nursing service is more humanized, the popularization of the nursing and disease prevention knowledge to the patients is more precise, and mental health care to the patients is well emphasized [12], so that the patients can get much more precise nursing care. Besides, all nursing staff have been trained, and passed the assessment of nursing work for ANVUGIB patients. They have fully mastered the skill about how to prevent complications and rebleeding related to ANVUGIB, so as to reduce the incidence of those kinds of situation. In the future, studies will be conducted to further determine the intervention effect of hierarchical nursing, such as rebleeding detection time, medical treatment (including endoscopy), time to antibiotics, and intensive care time for hemorrhagic shock. In addition, this study considered bleeding-related complications but did not evaluate the patient's condition and bleeding severity score. APACHE II and Forrest classification for UGI hemorrhage can be included in later studies to further explore the effectiveness of the clinical hierarchical nursing intervention to relieve the bleeding condition of patients.

\subsection{Clinical hierarchical nursing intervention can improve the quality of life of patients with ANVUGIB}

This study found that after nursing intervention, the scores of the observation group in eight dimensions of physiological function, physiological functioning, physical pain, general health, vitality, social function, mental health, and emotional function were significantly higher than those of the control group. The reported results from Qiong et al. [13] also proved that hierarchical nursing can significantly improve the patients' quality of life, which is similar to the results of this study. Because of acute massive hemorrhage, each body function of each function and the quality of life of the ANVUGIB patients reduced significantly. Routine nursing care has the disadvantages such as unclear job duty division, low-level nursing staff are insufficient in working skills and experience. The key to hierarchical nursing management lies in hierarchical training. Through hierarchical training of nurses at different levels, a talent training plan and a feasible management system are formulated, so as to gradually improve the professional skills of nurses, which has a significant effect on improving the quality of nursing. Relevant studies have confirmed that the application of hierarchical nursing management to respiratory and critical care department helps perform the management functions of nursing staff at different levels so as to provide better services for patients, and significantly improve the patients' quality of life.

\subsection{Clinical hierarchical nursing intervention can improve the medical compliance of the ANVUGIB patients}

The study found that although the medical compliance of the observation group was higher than that of the control group, no statistical difference was observed. This may be due to the reason that the research objects of this study is relatively less and concentrated in one hospital. Most of the ANVUGIB patients suffer from anxiety, fear, irritability and other adverse emotions due to the rapid onset and progression of the disease, and may even lose confidence in treatment and show resistance to clinical treatments. Routine nursing care lacks personalized and targeted nursing service, so the patients' feeling to the nursing service is relatively weak. While the hierarchical nursing intervention provides personalized and targeted medical care service. During the nursing process, 
sustained and effective nursing guidance and rationalized rehabilitation suggestions make the patients be more complied to the medical staff [14]. Therefore, the patients are in a positive state of mind to accept treatment, and the medical compliance is increased significantly.

\subsection{Clinical hierarchical nursing intervention can improve the disease knowledge mastery rate of the ANVUGIB patients}

ANVUGIB belongs to critical clinical disease, and there are much fewer people knowing about the disease. Routine nursing has disadvantage on popularization of the disease knowledge, whereas the hierarchical nursing not only provide disease knowledge education throughout the whole nursing process, but also take advantage of the communication effectiveness of the WeChat group to popularize the ANVUGIB related health knowledge to the ANVUGIB patients.

\subsection{Clinical hierarchical nursing intervention can improve the comfort level of the ANVUGIB patients}

This study confirmed that the nursing satisfaction of the observation group was $98.67 \%$, which was significantly higher than that of the control group $(89.33 \%)$. This study found that hierarchical nursing intervention can effectively improve the comfort level of patients. Due to the rapid onset and rapid progression of the disease, most ANVUGIB patients have unhealthy psychology such as anxiety and fear. Hierarchical nursing can pay close attention to the patient's psychological condition and provide individualized and targeted nursing measures to improve their comfort level and satisfaction. Compared with the conventional nursing mode, in the hierarchical nursing intervention mode, nurse-patient communication is more harmonious, and patients are more likely to feel the care of medical staff, which is worthy of clinical promotion in the future.

\section{Conclusions}

Taken together, clinical hierarchical nursing intervention can significantly reduce the incidence of complications and rebleeding of the ANVUGIB patients, and improve the patients' the quality of life, medical compliance, comfort level and nursing satisfaction. In the future, clinical practice should further explore the effectiveness of hierarchical nursing, and combine the ANVUGIB disease characteristics and the development of specialist nursing to establish a more efficient and scientific hierarchical nursing mode.

\section{AUTHOR CONTRIBUTIONS}

QS and YZ designed the study, supervised the data collection. TH analyzed the data, interpreted the data. TM prepare the manuscript for publication and reviewed the draft of the manuscript. All authors have read and approved the manuscript.

\section{ETHICS APPROVAL AND CONSENT TO PARTICIPATE}

All procedures performed in studies involving human participants were in accordance with the standards upheld by the Ethics Committee of the 904th Hospital of Joint Logistic Support Force, PLA and with those of the 1964 Helsinki Declaration and its later amendments for ethical research involving human subjects (Approval No. 2015-07).

\section{ACKNOWLEDGMENT}

Thanks to all the peer reviewers for their opinions and suggestions.

\section{FUNDING}

This research received no external funding.

\section{CONFLICT OF INTEREST}

The authors declare no conflict of interest.

\section{REFERENCES}

[1] Loffroy R, Mouillot T, Bardou M, Chevallier O. Current role of cyanoacrylate glue transcatheter embolization in the treatment of acute nonvariceal gastrointestinal bleeding. Expert Review of Gastroenterology \& Hepatology. 2020; 14: 975-984

[2] Sey MSL, Mohammed SB, Brahmania M, Singh S, Kahan BC, Jairath V. Comparative outcomes in patients with ulcer- vs non-ulcer-related acute upper gastrointestinal bleeding in the United Kingdom: a nationwide cohort of 4474 patients. Alimentary Pharmacology and Therapeutics. 2019; 49: 537-545.

[3] Bhutia KD, Lamtha SC. Retrospective study of etiology of non variceal acute gastrointestinal bleeding in Eastern Himalayan region of india in Sikkim. Journal of Family Medicine and Primary Care. 2019; 8: 573575.

[4] Chinese Journal of Internal Medicine; National Medical Journal of China; Chinese Journal of Digestion; Chinese Journal of Digestive Endoscopy; Chinese Digestive Endoscopist Association. Guidelines for the diagnosis and treatment of acute non-variceal upper gastrointestinal bleeding (2018, Hangzhou). Zhonghua Nei Ke Za Zhi. 2019; 58: 173-180. (In Chinese)

[5] Yang T, Feng C, Qu Y, Wang Q, Yang Y, Wang B, et al. Effect of teriparatide on quality of life in patients with postmenopausal osteoporosis: a retrospective cohort study. Journal of International Medical Research. 2020; 48: 030006051987674.

[6] Pandey V, Patil M, Patel R, Chaubal A, Ingle M, Shukla A. Prevalence of splenic vein thrombosis and risk of gastrointestinal bleeding in chronic pancreatitis patients attending a tertiary hospital in western India. Journal of Family Medicine and Primary Care. 2020; 8: 818-822.

[7] Jono F, Iida H, Fujita K, Kaai M, Kanoshima K, Ohkuma K, et al. Comparison of computed tomography findings with clinical risks factors for endoscopic therapy in upper gastrointestinal bleeding cases. Journal of Clinical Biochemistry and Nutrition. 2019; 65: 138-145.

[8] Wedi E, Fischer A, Hochberger J, Jung C, Orkut S, Richter-Schrag HJ. Multicenter evaluation of first-line endoscopic treatment with the OTSC in acute non-variceal upper gastrointestinal bleeding and comparison with the Rockall cohort: the FLETRock study. Surgical Endoscopy. 2018; 32: 307-314.

[9] Winslow SA, Fickley S, Knight D, Richards K, Rosson J, Rumbley N. Staff nurses revitalize a clinical ladder program through shared governance. Journal for Nurses in Staff Development. 2011; 27: 13-17.

[10] Paplanus LM, Bartley-Daniele P, Mitra KS. Knowledge translation: a Nurse Practitioner Clinical Ladder Advancement Program in a university- 
affiliated, integrated medical center. Journal of the American Association of Nurse Practitioners. 2014; 26: 424-437.

[11] Ko YK, Yu S. Clinical ladder program implementation: a project guide. Journal of Nursing Administration. 2014; 44: 612-616.

[12] Yue L, Plummer V, Cross W. The effectiveness of nurse education and training for clinical alarm response and management: a systematic review. Journal of Clinical Nursing. 2017; 26: 2511-2526.

[13] Wedi E, Fischer A, Hochberger J, Jung C, Orkut S, Richter-Schrag HJ. Multicenter evaluation of first-line endoscopic treatment with the OTSC in acute non-variceal upper gastrointestinal bleeding and comparison with the Rockall cohort: the FLETRock study. Surgical Endoscopy. 2018; 32: 307-314.
[14] Weng YH, Chen C, Kuo KN, Yang CY, Lo HL, Chen KH, et al. Implementation of evidence-based practice in relation to a clinical nursing ladder system: a national survey in Taiwan. Worldviews on EvidenceBased Nursing. 2015; 12: 22-30.

How to cite this article: Qin Shen, Yang Zheng, Ting He, Ting Mao. The effect of clinical hierarchical nursing intervention on patients with acute non-variceal upper gastrointestinal bleeding. Signa Vitae. 2021;17(3):144-150. doi:10.22514/sv.2021.076. 